\title{
Padrões de atividade física em diferentes domínios e ausência de diabetes em adultos
}

\author{
F.J.G. Pitanga, L.A.B. Almeida, M.M. Freitas, C.P.S. Pitanga, C.C. Beck
}

\begin{abstract}
O objetivo foi analisar os padrões de atividades físicas (caminhada, moderada e vigorosa) em diferentes domínios (trabalho, deslocamento, atividade doméstica e tempo livre) como preditores da ausência de diabetes. O estudo foi transversal realizado na cidade de Lauro de Freitas, Brasil, com 522 indivíduos maiores de 18 anos, $57.8 \%$ do sexo feminino. Foram construídas curvas Receiver Operating Characteristic (ROC) e comparadas às áreas entre os padrões de atividades físicas nos diversos domínios e a ausência de diabetes. Identificou-se também os pontos de corte da atividade física (minutos/semana) para predizer a ausência de diabetes. Foi utilizado o intervalo de confiança a 95\%. Encontrouse maiores áreas sob a curva ROC para a atividade física de tempo livre e para os diferentes domínios analisados conjuntamente. A caminhada não foi boa preditora da ausência de diabetes. Observou-se que atividades físicas acumuladas nos diferentes domínios realizadas por 185 minutos/semana em intensidade moderada, ou ainda, realizadas por 285 minutos/semana em intensidades de caminhada, moderada ou vigorosa foram os melhores pontos de corte para predizer a ausência de diabetes. A prática da atividade física, principalmente no tempo livre ou acumulada nos diferentes domínios, deve ser sugerida em padrões adequados visando contribuir para a prevenção da diabetes.

Palavras-chave: atividade física, diabetes, curvas ROC
\end{abstract}

\section{Patterns of physical activity in different domains and diabetes absence in adults}

The objective was to analyse the patterns of physical activity (moderate and vigorous walking) in different domains (work, commuting, household, leisure time) as predictors of absence of diabetes. This was a cross-sectional study in the city of Lauro de Freitas, Bahia, Brazil, with a sample of 522 individuals, $57.8 \%$ of them female, over 18 years of age. Receiver Operating Characteristic curves (ROC) were constructed and compared to patterns of physical activity in differing domains and absence of diabetes. Cut-off points of physical activity (minutes/week) were established to predict the absence of diabetes. A 95\% confidence interval was considered to find the largest areas under the ROC curves for leisure time physical activity and for the differing domains analysed altogether. Walking was not a good predictor of diabetes absence. Accumulated physical activity in different domains (185 minutes/week of moderate intensity or 285 minutes/week of moderate or vigorous intensity walking) presented the best cut-off points for predicting the absence of diabetes. Practicing physical activity, especially leisure time or accumulated in different domains, contributes to diabetes prevention.

Keywords: physical activity, diabetes, ROC curves

Francisco José Pitanga. Faculdade de Educação da Universidade Federal da Bahia, Brasil.

Luiz Alberto Almeida. Laboratório de Biociência da Motricidade Humana da Universidade Castelo Branco; Universidade Estadual de Feira de Santana, Brasil.

Marcela Mota Freitas e Cristiano Penas Pitanga. União Metropolitana de Ensino, Brasil.

Carmem Cristina Beck. Centro de Desportos da Universidade Federal de Santa Catarina, Brasil.

Endereço para correspondência: Francisco José Pitanga, Faculdade de Educação, Universidade Federal da

Bahia, Av. Reitor Miguel Calmon, s/n, CEP: 40110-100, Salvador, Bahia - Brasil.

E-mail: pitanga@lognet.com.br 
O diabetes é um grave problema de saúde pública e um dos importantes fatores de risco para doenças cardiovasculares. Atualmente, observa-se aumento da sua ocorrência em várias regiões do mundo com projeção de atingir 300 milhões de pessoas no ano 2030 (Wlid, Roglic, Gree, Sicree, \& King, 2004).

A atividade física definida como qualquer movimento corporal produzido pela musculatura esquelética que resulte em gasto energético acima dos níveis de repouso (Caspersen, Powell, \& Christenson, 1985) é composta pelos domínios das atividades de trabalho, deslocamentos, atividades domésticas e tempo livre.

As evidências da associação inversa entre atividade física e diabetes são observadas em diversos estudos ( $\mathrm{Hu}$ et al., 2001, 2003, 2004), porém o poder preditivo e os pontos de corte dos padrões de atividade física (caminhada, moderada e vigorosa) em seus diferentes domínios (trabalho, deslocamentos, atividades domésticas e tempo livre) para prevenção do diabetes permanecem especulativos.

A atividade física regular ajuda a diminuir e/ou manter o peso corporal, a reduzir a necessidade de antidiabéticos orais, a diminuir a resistência à insulina e contribui para melhora do controle glicêmico, o que, por sua vez, reduz o risco das complicações associadas ao diabetes (Ford \& Herman, 1995).

A identificação dos domínios e dos padrões de atividade física, mais adequados, para a prevenção e o tratamento do diabetes melittus pode direcionar e otimizar os benefícios dos programas de práticas corporais. Além disso, é necessário também, conhecer os pontos de corte dos diferentes domínios e padrões da atividade física, em minutos por semana, para que esses benefícios sejam maximizados.

Assim, o objetivo desse estudo foi analisar o poder preditivo e identificar os pontos de corte dos padrões de atividades físicas, em seus diferentes domínios, para a ausência de diabetes em adultos de ambos os sexos.

\section{MÉTODO}

Trata-se de estudo de corte transversal realizado no município de Lauro de Freitas situado no nordeste do estado da Bahia, região Metropolitana de Salvador (Brasil). Com extensão territorial de 59 quilômetros quadrados, o município de Lauro de Freitas possuía índice de desenvolvimento humano (IDH) de 0.771 e produto interno bruto (PIB) per capita $\mathrm{R} \$$ de 12046.00 . De acordo com o Censo Demográfico de 2005 sua população estimada era de 138240 habitantes (Datasus, 2005).

\section{Amostra}

O cálculo da amostra foi baseado em Kish (1965) levando-se em consideração os seguintes parâmetros: tamanho da população de 138240 habitantes, prevalência de inatividade física de $50 \%$ tendo como base o estudo realizado no Estado de São Paulo - Brasil (Matsudo et al., 2002), bem como da maior prevalência entre as variáveis analisados no estudo, nível de confiança de $95 \%$ de precisão e erro assumido para a prevalência esperada de $5 \%$. 
A amostra estimada foi de 500 adultos com idade maior ou igual a 18 anos, ampliada para 600, prevendo eventuais perdas e recusas.

A amostra foi probabilística, em múltiplos estágios e por conglomerados de classes sociais informados pela Secretaria de Ação Social da Prefeitura da cidade de Lauro de Freitas a partir do poder aquisitivo dos moradores dos bairros. Considerou-se como classe $\mathrm{A}$ (alta e média alta), classe B (média), classe C (média e baixa) e classe D (baixa e pobreza).

Inicialmente o mapa da cidade foi dividido em microrregiões de acordo com a classe social predominante. Em seguida foram sorteadas 25 ruas da cidade de Lauro de Freitas pertencentes aos quatro níveis sociais (Classe A, B, C, D). A divisão das ruas foi proporcional ao nível sócio-econômico e obedeceu ao seguinte quantitativo: seis ruas em cada um dos conglomerados A, B e $\mathrm{C}$ e sete ruas na classe D. Em cada rua treze domicílios foram sorteados por amostra sistemática. O intervalo entre as casas variou de acordo com a quantidade de domicílios encontrados em cada rua. A cada residência visitada foram sorteados dois indivíduos adultos (um do sexo masculino e outro do sexo feminino). Foram excluídas as residências desabitadas, as pessoas que se recusaram a responder o questionário e os indivíduos acamadas por problemas de saúde.

Apesar de a metodologia ter sido desenvolvida para obtenção de amostra com igual número de homens e mulheres, isso não aconteceu, devido ao fato de que nem sempre o homem estava presente no domicílio por motivos de trabalho e atividades da vida diária.

Desta forma, ao final da coleta de dados a amostra ficou constituída de 522 pessoas, 220 do sexo masculino e 302 do sexo feminino.

Todos os participantes da pesquisa assinaram o Termo de Participação Livre e Esclarecida e foram entrevistados em domicílio.

\section{Coleta de dados}

Cinco avaliadores foram devidamente preparados e treinados para todas as etapas do trabalho. O índice de confiabilidade inter-avaliadores foi testado para aplicação do IPAQ por meio do coeficiente Kappa, o qual apresentou bom índice de concordância (0.61) (Pereira, 1995). O erro técnico de medidas para o peso e a estatura foi considerado baixo (1.2\%) (Gore et al., 2005).

Os dados sócio-demográficos e os fatores relacionados à saúde foram coletados por meio de questionário. Para obtenção dos dados antropométricos utilizou-se os seguintes protocolos: a estatura foi mensurada com uma trena antropométrica em aço Sanny (Brasil), com os indivíduos descalços, em posição ereta, com pés e calcanhares unidos e encostados na parede, braços estendidos ao longo do corpo, respiração normal seguindo o Plano de Frankfurt (Pitanga, 2006).

O peso foi mensurado duas vezes utilizando-se balanças da marca Plenna com precisão de 100 gramas, todas aferidas previamente pelo Instituto Nacional de Metrologia (INMETRO). Ao entrevistado era solicitado que 
subisse na balança com os pés descalços, com o mínimo de roupa possível (Pitanga, 2006).

Para identificar os padrões e domínios da atividade física o instrumento utilizado foi o International Physical Activity Questionnary (IPAQ) versão longa, constituído de questões relativas à frequência e duração de atividades físicas (caminhada, moderada e vigorosa) desenvolvidas no trabalho, no deslocamento, nas atividades domésticas e no tempo livre (Matsudo et al., 2001).

Os valores de cada um dos padrões de atividades físicas, em seus diferentes domínios, foram relatados em minutos/semana por meio da multiplicação da frequência semanal pela duração de cada uma das atividades realizadas.

\section{Análise estatística}

A caracterização das variáveis foi apresentada em média, desvio padrão, valores mínimos, máximos e frequências.

Para comparar a distribuição das variáveis segundo os sexos utilizou-se o teste " $\mathrm{t}$ " de Student para amostras independentes (dados contínuos) e o teste qui-quadrado (dados categóricos).

O poder preditivo e os pontos de corte dos diferentes padrões e domínios da atividade física para ausência de diabetes foram identificados através das curvas Receiver Operating Characteristic (ROC), frequentemente utilizadas para determinação de pontos de corte em testes diagnósticos ou de triagem (Erdreich \& Lee, 1981).

Inicialmente foi identificada a área total sob a curva ROC entre os padrões de atividade física (caminhada, mode- rada e vigorosa) em seus diferentes domínios (trabalho, deslocamento, atividade doméstica, tempo livre) e atividade física total (quatro domínios analisados conjuntamente) para a ausência de diabetes. Quanto maior área sob a curva ROC, maior o poder discriminatório da atividade física para ausência de diabetes.

Utilizou-se intervalo de confiança (IC) a $95 \%$, o qual determina se a capacidade preditiva dos padrões de atividade física em seus diferentes domínios não é devido ao acaso, não devendo o seu limite inferior ser menor do que 0.50 (Schisterman, Faraggi, Reiser, \& Trevisan, 2001).

$\mathrm{Na}$ sequência, foram calculadas a sensibilidade e especificidade, além dos pontos de corte para os padrões de atividades físicas (caminhada, moderada e vigorosa) em seus diferentes domínios (trabalho, deslocamento, atividade doméstica, tempo livre) e atividade física total para a ausência de diabetes.

Valores identificados por intermédio da curva ROC constituem-se em pontos de corte que deverão promover um mais adequado equilíbrio entre sensibilidade e especificidade para atividade física como discriminador da ausência de diabetes.

Os dados foram analisados através do programa estatístico STATA, versão 7.0.

O presente estudo foi aprovado pelo Comitê de Ética da Faculdade Adventista de Fisioterapia (FAFIS) localizada na cidade de Cachoeira, Bahia, Brasil segundo o parecer número 0033/2007. 


\section{RESULTADOS}

As características da amostra estão demonstradas na tabela 1 . Observa-se que os homens são mais pesados e mais altos do que as mulheres. Quanto à idade não existem diferenças entre os sexos.

Tabela 1

Média, desvio padrão, valor mínimo, máximo e frequência das variáveis analisadas no estudo

\begin{tabular}{lccc}
\hline \multicolumn{1}{c}{ Variáveis } & Masculino $(n=220)$ & Feminino $(n=302)$ & $p$ \\
\hline \multirow{2}{*}{ Idade (anos) } & $40.2 \pm 15.3$ & $40.4 \pm 14.7$ & $.88^{*}$ \\
& $(18-85)$ & $(20-86)$ & \\
Peso $(\mathrm{kg})$ & $75.0 \pm 14.0$ & $68.0 \pm 14.4$ & $.00^{*}$ \\
& $(41-126)$ & $(43-129)$ & $.00^{*}$
\end{tabular}

AF no trabalho $(\mathrm{min} / \mathrm{sem})$

$\begin{array}{lc}\text { Caminhada } & 208.2 \pm 487.7 \\ & (0-2880) \\ \text { Moderada } & 142.8 \pm 448.6 \\ & (0-2940) \\ \text { Vigorosa } & 44.2 \pm 156.6 \\ & (0-1080) \\ \text { Total } & 395.5 \pm 857.8 \\ & (0-5310)\end{array}$
$88.0 \pm 342.4$
(0-2880)
$.00^{*}$

$49.3 \pm 258.1$

(0-2880)

$.00^{*}$

$14.7 \pm 122.0$

(0-2000)

$.01^{*}$

$152.0 \pm 517.9$

$(0-5360)$

$.00^{*}$

AF no deslocamento $(\mathrm{min} / \mathrm{sem})$

Caminhada

$$
\begin{gathered}
99.4 \pm 189.1 \\
(0-1440) \\
52.8 \pm 126.6 \\
(0-840) \\
152.2 \pm 239.1 \\
(0-1620)
\end{gathered}
$$

$142.7 \pm 300.3$

$$
\text { (0-3360) }
$$

$6.1 \pm 63.8$

Bicicleta

(0-1050)

$.00^{*}$

Total

$148.8 \pm 304.8$

(0-3360)

$.89^{*}$

AF doméstica $(\mathrm{min} / \mathrm{sem})$

Moderada em casa

$105.8 \pm 277.6$

(0-1890)

$420.5 \pm 635.7$

(0-4200)

$.00^{*}$

$112.4 \pm 300.4$

(0-2400)

$149.4 \pm 302.4$

(0-2400)

$.17^{*}$

$39.2 \pm 171.0$

Vigorosa no quintal

(0-1680)

$59.9 \pm 139.8$

$$
(0-720)
$$

$.13^{*}$

Total

$257.4 \pm 514.3$

(0-4200)

$629.8 \pm 766.6$

(0-4630)

$.00^{*}$ 
10 | F.J.G. Pitanga, L.A.B. Almeida, M.M. Freitas, C.P.S. Pitanga, C.C. Beck

Tabela 1 (continuação)

Média, desvio padrão, valor mínimo, máximo e frequência das variáveis analisadas no estudo

\begin{tabular}{|c|c|c|c|}
\hline Variáveis & Masculino $(n=220)$ & Feminino $(n=302)$ & $p$ \\
\hline \multicolumn{4}{|c|}{ AF no tempo livre $(\mathrm{min} / \mathrm{sem})$} \\
\hline Caminhada & $\begin{array}{c}46.5 \pm 114.9 \\
(0-700)\end{array}$ & $\begin{array}{c}34.1 \pm 88.9 \\
(0-450)\end{array}$ & $.16^{*}$ \\
\hline Moderada & $\begin{array}{c}84.2 \pm 215.5 \\
(0-1680)\end{array}$ & $\begin{array}{c}18.9 \pm 85.3 \\
(0-840)\end{array}$ & $.00^{*}$ \\
\hline Vigorosa & $\begin{array}{c}31.0 \pm 95.3 \\
(0-720)\end{array}$ & $\begin{array}{c}7.7 \pm 54.8 \\
(0-720)\end{array}$ & $.00^{*}$ \\
\hline Total & $\begin{array}{c}161.7 \pm 285.1 \\
(0-1710)\end{array}$ & $\begin{array}{c}60.8 \pm 142.7 \\
(0-840)\end{array}$ & $.00^{*}$ \\
\hline \multicolumn{4}{|l|}{ AF total $(\mathrm{min} / \mathrm{sem})$} \\
\hline Caminhada & $\begin{array}{c}354.1 \pm 545.2 \\
(0-2940)\end{array}$ & $\begin{array}{c}264.9 \pm 465.9 \\
(0-3360)\end{array}$ & $.04^{*}$ \\
\hline Moderada & $\begin{array}{c}497.9 \pm 711.2 \\
(0-4535)\end{array}$ & $\begin{array}{c}644.2 \pm 786.8 \\
(0-4360)\end{array}$ & $.03^{*}$ \\
\hline Vigorosa & $\begin{array}{c}114.7 \pm 242.8 \\
(0-1680)\end{array}$ & $\begin{array}{c}82.3 \pm 199.0 \\
(0-2300)\end{array}$ & $.09^{*}$ \\
\hline Total & $\begin{array}{c}966.8 \pm 1105.5 \\
(0-7250)\end{array}$ & $\begin{array}{c}991.4 \pm 1060.0 \\
(0-8220)\end{array}$ & $.79 *$ \\
\hline \multicolumn{4}{|l|}{ Diabetes (\%) } \\
\hline Presença & 4.5 & 3.3 & \multirow{2}{*}{$.31^{* *}$} \\
\hline Ausência & 95.5 & 96.7 & \\
\hline
\end{tabular}

Nota: AF = Atividade Física; * Valor obtido através do teste "t" de student para amostras independentes; ** Valor obtido através do teste qui-quadrado; AF total = soma dos domínios trabalho, deslocamento, doméstica e no tempo livre

Com relação aos domínios da atividade física os homens são mais ativos no trabalho e no tempo livre, enquanto as mulheres são mais ativas nas atividades domésticas. No deslocamento e na atividade física total (considerando os quatro domínios) não existem diferenças entre homens e mulheres. Quanto aos padrões de atividades físicas (considerando-se os quatro domínios) observa-se que os homens são mais ativos na caminhada enquanto as mulheres são mais ativas nas atividades moderadas. Não existem diferenças entre homens e mulheres nas atividades vigorosas. Observa-se, também, que não existem diferenças entre os sexos quanto à presença ou ausência de diabetes.

$\mathrm{Na}$ tabela 2 pode-se observar as áreas sob as curvas ROC, com seus respectivos intervalos de confiança, dos padrões de atividades físicas em seus diferentes domínios como preditores da ausência de diabetes. Foram construídas curvas ROC para o sexo masculino, feminino e para ambos os sexos. De modo geral, as maiores áreas são 
observadas no domínio da atividade física de tempo livre. Observa-se também que na caminhada as áreas sob as curvas ROC não foram significativas em nenhum dos domínios analisados.

Tabela 2

Áreas sob a curva ROC e IC95\% entre os padrões de atividades física em seus diferentes domínios como preditores da ausência de diabetes em adultos de ambos os sexos

\begin{tabular}{|c|c|c|c|}
\hline Domínios & Ambos os sexos & Masculino & Feminino \\
\hline \multicolumn{4}{|l|}{ AF no tempo livre } \\
\hline Caminhada & $.54(.46-.62)$ & $.53(.40-.66)$ & $.55(.45-.65)$ \\
\hline Moderada & $.58(.53-.63) *$ & $.64(.55-.72) *$ & $.54(.53-.56) *$ \\
\hline Vigorosa & $.55(.54-.56) *$ & $.59(.57-.62) *$ & $.52(.51-.53) *$ \\
\hline Total & $.62(.54-.70) *$ & $.68(.56-.80) *$ & $.58(.42-.67)$ \\
\hline \multicolumn{4}{|l|}{ AF no trabalho } \\
\hline Caminhada & $.54(.44-.64)$ & $.54(.37-.71)$ & $.55(.44-.66)$ \\
\hline Moderada & $.53(.45-.61)$ & $.51(.37-.65)$ & $.57(.55-.59) *$ \\
\hline Vigorosa & $.54(.53-.56) *$ & $.57(.55-.60) *$ & $.52(.51-.54) *$ \\
\hline Total & $.57(.48-.67)$ & $.59(.42-.77)$ & $.58(.47-.68)$ \\
\hline \multicolumn{4}{|l|}{ AF no deslocamento } \\
\hline Caminhada & $.60(.49-.72)$ & $.55(.39-.70)$ & $.64(.46-.82)$ \\
\hline Bicicleta & $.53(.46-.59)$ & $.62(.52-.71) *$ & $.46(.36-.56)$ \\
\hline Total & $.60(.50-.72) *$ & $.62(.50-.74) *$ & $.59(.41-.77)$ \\
\hline \multicolumn{4}{|l|}{ AF doméstica } \\
\hline Moderada em casa & $.62(.52-.73) *$ & $.51(.35-.67)$ & $.70(.56-.83) *$ \\
\hline Moderada no quintal & $.50(.37-.63)$ & $.51(.35-.66)$ & $.46(.24-.66)$ \\
\hline Vigorosa no quintal & $.50(.41-.59)$ & $.52(.43-.61)$ & $.47(.32-.62)$ \\
\hline Total & $.60(.48-.71)$ & $.56(.41-.71)$ & $.60(.44-.75)$ \\
\hline
\end{tabular}

Nota: $\mathrm{AF}=$ Atividade Física; $\mathrm{ROC}=$ Receiver Operating Characteristic; IC95\% = intervalo de confiança a 95\%; * Área sob a curva ROC apresentando poder discriminatório para ausência de diabetes $(\mathrm{Li}-\mathrm{IC} \geq .50)$

Tabela 3

Áreas sob a curva ROC e IC95\% entre os padrões de atividade física total como preditores da ausência de diabetes em adultos

\begin{tabular}{cccc}
\hline Padrões de AF & Ambos os sexos & Masculino & Feminino \\
\hline Caminhada & $.62(.48-.75)$ & $.56(.38-.73)$ & $.69(.49-.88)$ \\
Moderada & $.64(.53-.75)^{*}$ & $.63(.48-.78)$ & $.62(.47-.77)$ \\
Vigorosa & $.59(.51-.68)^{*}$ & $.67(.60-.74) *$ & $.51(.37-.66)$ \\
Total & $.68(.57-.80)^{*}$ & $.67(.49-.85)$ & $.69(.54-.84)^{*}$ \\
\hline
\end{tabular}

Nota: AF = Atividade Física = soma dos domínios trabalho, deslocamento, doméstica e no tempo livre; ROC $=$ Receiver Operating Characteristic; IC95\% = intervalo de confiança a 95\%; * Área sob a curva ROC apresentando poder discriminatório para ausência de diabetes ( $\mathrm{Li}-\mathrm{IC} \geq .50)$ 
Na tabela 3 e figura 1 observam-se as áreas sob as curvas ROC, com seus respectivos intervalos de confiança, entre os padrões da atividade física total (quatro domínios) como preditores da ausência de diabetes para homens, mulheres e ambos os sexos. As maiores áreas são observadas quando homens e mulheres são analisados conjuntamente. Mais uma vez observase que na caminhada as áreas sob as curvas ROC não foram significativas.

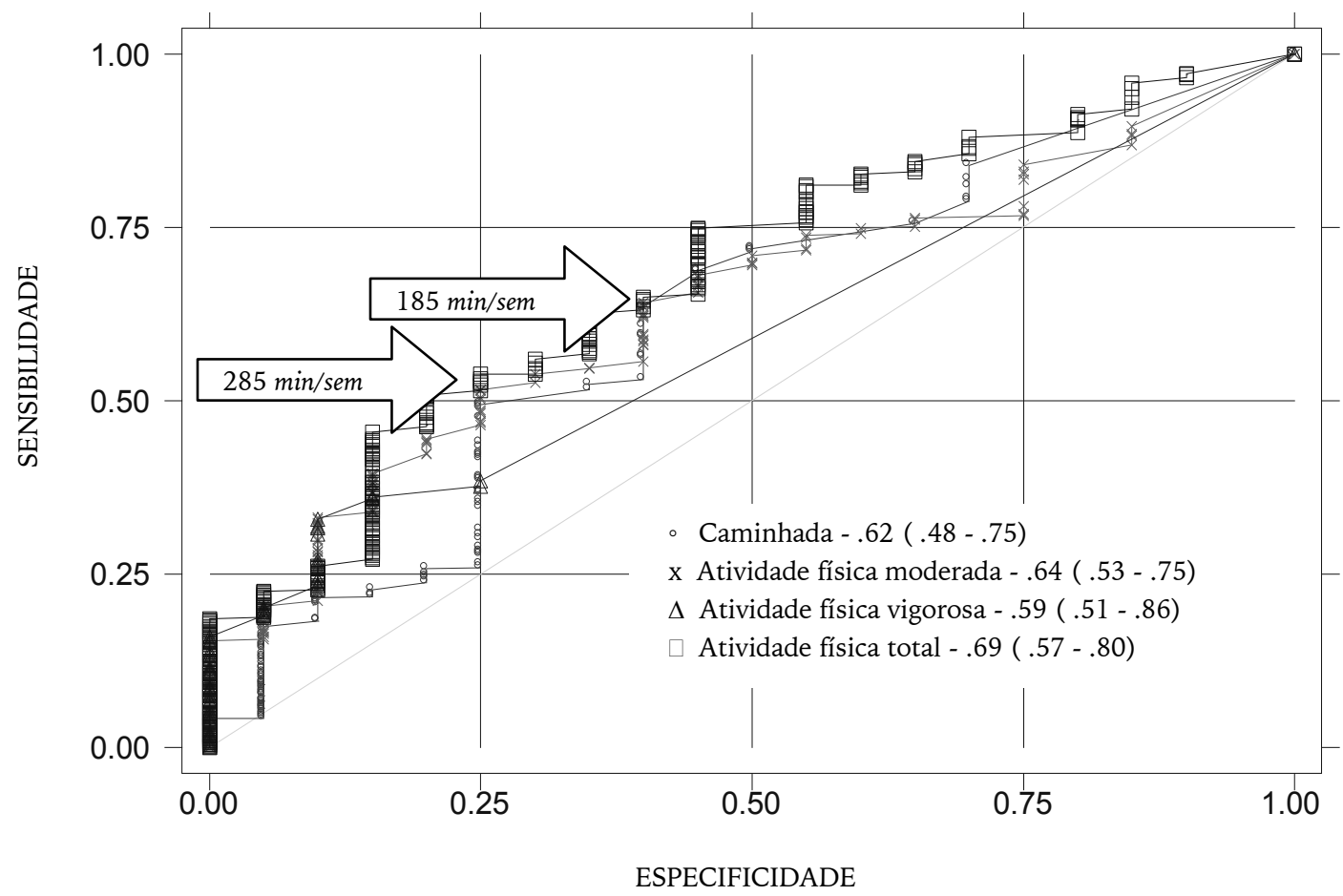

Figura 1. Áreas sob a curva ROC, com seus respectivos pontos de corte para atividade física moderada e total, entre os padrões e domínios da atividade física total como preditores da ausência de diabetes em homens e mulheres analisados conjuntamente

A tabela 4 apresenta os pontos de corte, com suas respectivas sensibilidades e especificidades, dos padrões da atividade física total (trabalho, deslocamentos, atividades domésticas e tempo livre) como preditores da ausência de diabetes em homens e mulheres analisados conjuntamente. Os pontos de corte foram determinados para a atividade física moderada (185 min/semana) e para o total de atividade física (caminhada + moderada + vigorosa) (285 min/semana) (figura 1). Considerando-se separadamente cada um dos domínios da atividade com seus respectivos padrões, não foi possível a determinação dos pontos de corte em virtude das áreas sob as curvas ROC não serem significativas e/ou os valores de sensibilidade e especificidade não serem adequados. 
Tabela 4

Pontos de corte, sensibilidade e especificidade dos padrões da atividade física total como preditores da ausência de diabetes - homens e mulheres analisados conjuntamente

\begin{tabular}{cccc}
\hline Padrões de AF & $\begin{array}{c}\text { Ponto de corte } \\
\text { (minutos/semana) }\end{array}$ & $\begin{array}{c}\text { Sensibilidade } \\
(\%)\end{array}$ & $\begin{array}{c}\text { Especificidade } \\
(\%)\end{array}$ \\
\hline Caminhada & NR & - & - \\
Moderada & 185 & 64.1 & 60.0 \\
Vigorosa & NR & - & - \\
Total & 285 & 74.9 & 55.0 \\
\hline
\end{tabular}

Nota: $\mathrm{AF}=$ Atividade Física = soma dos domínios trabalho, deslocamento, doméstica e no tempo livre; $\mathrm{NR}$ = indicador não recomendável para a predição de "ausência" de diabetes (pontos de corte não apresentam boas sensibilidade e especificidade)

\section{DISCUSSÃO}

O estudo demonstra o poder preditivo dos padrões de atividades físicas (caminhada, moderada e vigorosa) em seus diferentes domínios (trabalho, deslocamento, atividades domésticas, tempo livre) e atividade física total para a ausência de diabetes. Além disso, identifica os pontos de corte (minutos por semana), da atividade física total (quatro domínios), por meio dos valores que apresentam maior equilíbrio entre a sensibilidade e a especificidade para discriminar a ausência de diabetes.

Uma possível limitação do estudo foi a determinação do diabetes autorreferido que pode ter provocado subestimação na prevalência desta variável, considerando que muitas pessoas podem desconhecer sua condição de diabético. Outra limitação pode ser atribuída à dificuldade na coleta de dados nos bairros de classes C e D por questões de focos de violência.

No presente estudo observou-se que os homens são mais ativos no trabalho e no tempo livre enquanto as mulheres são mais ativas nas atividades domésticas. No domínio deslocamento não observou-se diferenças entre os sexos, considerando que os homens deslocam-se mais de bicicleta e as mulheres deslocam-se mais caminhando fazendo com que nesse domínio a quantidade de atividade física realizada seja similar. Resultados contrários foram observados em estudo conduzido por Barengo, Kastarinen, Lakka, Nissinen e Tuomilehto (2006) quando relataram níveis parecidos de atividade física entre homens e mulheres no tempo livre e no trabalho. Com relação às atividades de deslocamento, os mesmos autores observaram que apenas $27 \%$ dos homens e $42 \%$ das mulheres atingiram mais de 15 minutos diários de deslocamento a pé ou de bicicleta.

Diversos autores têm demonstrado que a atividade física pode proporcionar benefícios na prevenção e tratamento do diabetes (Sigal, Kenny, Wasserman, \& Castaneda-Sceppa, 2004; Hayes \& Kriska, 2008), porém poucos trabalhos tentaram identificar o poder preditivo dos padrões de atividades físicas em seus diferentes domínios como discriminador da ausência de diabetes.

No nosso trabalho observou-se que a atividade física total, considerando-se 
os quatro domínios, e o domínio tempo livre mostraram-se os melhores preditores da ausência de diabetes. Os domínios das atividades físicas no trabalho e no deslocamento apresentaramse razoáveis, enquanto a atividade doméstica foi o domínio menos adequado para proteção de diabetes. Resultados parecidos com relação ao tempo livre e ao trabalho foram observados em recente publicação (Fretts et al., 2009), quando foi analisada a associação entre atividade física em 1651 índios americanos. Os resultados indicaram que a participação em qualquer nível de atividade física seria fator de proteção para a diabetes.

Outro aspecto que chama atenção no presente estudo é o fato da caminhada não se apresentar como preditora da ausência de diabetes em nenhum dos domínios de atividade física analisados. Uma possível explicação para este achado seria a utilização do IPAQ como instrumento de mensuração da atividade física, o qual não identifica a intensidade da caminhada realizada. Resultados contrários foram observados em 11.073 homens japoneses (Sato et al., 2007) onde a caminhada para o trabalho, com duração maior do que 21 minutos por dia, reduziu o risco de diabetes.

Por outro lado, as atividades moderadas e vigorosas nos diferentes domínios apresentaram-se satisfatórias para discriminar a ausência de diabetes, principalmente no domínio das atividades físicas de tempo livre. Concordando com nossos resultados, $\mathrm{Hu}$ et al. (2004), após estudo com acompanhamento de 3316 finlandeses de ambos os sexos, portadores de diabetes tipo 2 , observaram que a atividade física moderada ou vigorosa reduz a mortalidade cardiovascular em diabéticos. Deste modo, parece que realizar atividades físicas de intensidade moderada à vigorosa reduz a incidência de diabetes e previne complicações cardiovasculares associadas à diabetes.

Ainda na Filândia (Hu et al., 2003) foram acompanhados durante doze anos, 6898 homens e 7392 mulheres com objetivo de identificar qual dos padrões e domínios da atividade física proporcionaria a redução do risco de diabetes. Como resultado estes pesquisadores verificaram que as atividades físicas moderadas ou vigorosas realizadas no trabalho, no deslocamento ou no tempo livre reduziram de maneira significativa o risco de diabetes na população.

No presente estudo optou-se, também, por identificar os pontos de corte (minutos por semana) dos padrões de atividades físicas nos seus diferentes domínios para a ausência de diabetes. Observou-se que atividades físicas acumuladas nos diferentes domínios realizadas por 185 minutos/semana em intensidade moderada, ou ainda, realizadas por 285 minutos/semana em intensidades de caminhada, moderada ou vigorosa foram os melhores pontos de corte para predizer a ausência de diabetes. Quando os domínios da atividade física foram analisados individualmente os pontos de corte não puderam ser identificados porque as áreas sob as curvas ROC não foram significativas e/ou os valores de sensi- 
bilidade e especificidade não foram adequados.

A preocupação dos pesquisadores em identificar a quantidade de atividade física mais adequada para proporcionar benefícios à saúde não é recente. Blair, Kohl, Gordon e Paffenbarger (1992) buscaram identificar a dose de atividade física que proporcionaria efeitos mais significativos para proteção de diversos agravos à saúde. Nessa época foi sugerido que indivíduos adultos deveriam acumular pelo menos 30 minutos de caminhada por dia para obter benefícios mais significativos à saúde.

Em 1995, duas instituições de renomada competência internacional, o Centers for Disease Control and Prevention (CDC) e o American College of Sports Medicine (ACSM) (Pate et al., 1995) recomendaram que para adquirir proteção para agravos metabólicos e cardiovasculares, os adultos deveriam acumular trinta minutos de atividades físicas em intensidade moderada na maioria dos dias da semana.

Em recente diretriz publicada no Canadá (Warburton, Katzmarzyk, Rhodes, \& Shephard, 2007) sobre atividade física para adultos sugeriu-se que para redução do risco de diversas condições crônicas, particularmente doença arterial coronariana, hipertensão arterial e diabetes é necessário atividade física de intensidade moderada todos os dias da semana.

Os resultados deste estudo indicaram que a atividade física realizada no tempo livre e a atividade física total (tempo livre, trabalho, doméstica e deslocamento analisados conjuntamente) melhor predizem a ausência de diabetes. Com relação à quantidade necessária sugere-se que, atividades físicas acumuladas nos quatro domínios, e realizadas de $185 \mathrm{~min} / \mathrm{sem}$ em intensidade moderada ou $285 \mathrm{~min} / \mathrm{sem}$ de caminhada, mais atividade física moderada e vigorosa seriam as mais indicadas para previnir o diabetes. Observase também que, provavelmente, a caminhada isoladamente não seria uma boa estratégia para prevenção da diabetes. São necessários novos estudos que identifiquem a intensidade e o volume de atividades físicas mais adequados para a proteção de outros agravos metabólicos e cardiovasculares.

\section{REFERÊNCIAS}

Barengo, N. C., Kastarinen, M., Lakka, T., Nissinen. A., \& Tuomilehto, J. (2006). Different forms of physical activity and cardiovascular risk factors among 2464-year-old men and women in Finland. European Journal of Cardiovascular Prevention \& Rehabilitation, 13(1), 51-59.

Blair, S. N., Kohl, H. W., Gordon, N. F., \& Paffenbarger, R. S. Jr. (1992). How much physical activity is good for health? Annual Review of Public Health, 13, 99-126.

Caspersen, C. J., Powell, K. E., \& Christenson, G. M. (1985). Physical activity, exercise and physical fitness. Public Health Reports, 100, 126-131.

DATASUS (2005). Dados censitários dos municípios brasileiros. Lauro de Freitas: Ministério da Saúde. Acedido em Março, 6 de 2007 a partir de http://www.datasus.gov.br

Erdreich, L. S., \& Lee, E. T. (1981) Use of relative operating characteristics analysis in epidemiology: a method for dealing with subjective judgment. Ameri- 
can Journal of Epidemiology, 114(5), 649662.

Ford, E. S., \& Herman, W. H. (1995) Leisure time physical activity patterns in the U.S. diabetic population. Diabetes Care, 18, 27-33.

Fretts, A. M., Howard, B. V., Kriska, A. M., Smith, N. L., Lumley, T., Lee, E. T., et al. (2009). Physical activity and incident diabetes in american indians: The Strong Heart Study. American Journal of Epidemiology, 170, 632-639.

Gore, C., Norton, K., Olds, T., Whittingham, N., Birchall, K., Clough, M. et al. (2005). Erros de medição em antropometria. In K. Norton \& T. Olds (Eds.), Antropométrica (pp. 375-391). Porto Alegre: Artmed.

Hayes, C., \& Kriska, A. (2008). Role of physical activity in diabetes management and prevention. Journal of the American Dietetic Association, 108, S19S23.

Hu, F. B., Leitzmann, M. F., Stampfer, M. J., Colditz, G. A., Willett, W. C. \& Rimm, E. B. (2001). Physical activity and television watching in relation to risk for type 2 diabetes mellitus in men. Archives Internal Medicine, 161,1542-1548.

Hu, G., Qiao, Q., Silventoinen, K., Eriksson, J. G., Jousilahti, P., Lindstrom J., et al. (2003). Occupational, commuting, and leisure-time physical activity in relation to risk for type 2 diabetes in middle-aged Finnish men and women. Diabetologia, 46, 322-329.

Hu, G., Eriksson, J., Barengo, N. C., Lakka, T. A., Valle, T. T., Nissinen, A., et al. (2004). Occupational, commuting, and leisure-time physical activity in relation to total and cardiovascular mortality among finnish subjects with type 2 diabetes. Circulation, 110, 666-673.
Kish, L. (1965). Survey sampling. New York: John Wiley and Sons.

Matsudo, S., Araújo, T., Matsudo, V., Andrade, D., Andrade, E., Oliveira, L. C., et al (2001). Questionário internacional de atividade física (IPAQ): Estudo de validade e reprodutibilidade no Brasil. Revista Brasileira de Atividade Física e Saúde, 6(2), 5-18.

Matsudo, S. M., Matsudo, V. R., Araújo, T., Andrade, D., Andrade, E., Oliveira, L. C., et al. (2002). Nível de atividade física da população do estado de São Paulo: Análise de acordo com o gênero, idade e nível sócio-econômico, distribuição geográfica e de conhecimento. Revista Brasileira de Ciência e Movimento, 10(4), 41-50.

Pate, R. R., Pratt, M., Blair, S. N., Haskell, W. L., Macera, C. A, Bouchard, C., et al. (1995). Physical activity and public health: A recommendation from the Centers for Disease Control and Prevention and the American College of Sports Medicine. Journal of American Medical Association, 273, 402-407.

Pereira, M. (1995). Epidemiologia: Teoria e prática. Rio de Janeiro: Guanabara Koogan.

Pitanga, F. J. G. (2006). Teste, medidas e avaliação em educação física ( $5^{\mathrm{a}}$ ed.). São Paulo: Editora Phorte.

Sato, K. K., Hayashi, T., Kambe, H., Nakamura, Y., Harita, N., Endo, G., et al . (2007). Walking to work is an independent predictor of incidence of type 2 diabetes in Japanese men. Diabetes Care, 30(9), 2296-2298.

Schisterman, E. F., Faraggi, D., Reiser, B., \& Trevisan, M. (2001). Statistical inference for the area under the receiver operating characteristic curve in the presence of random measurement error. American Journal of Epidemiology, 154(2), 174-179. 
Sigal, R., Kenny, G., Wasserman, D., \& Castaneda-Sceppa, C. (2004). Physical activity/exercise and type 2 diabetes. Diabetes Care, 27(2), 2518-2539.

Warburton, D. E., Katzmarzyk, P. T., Rhodes, R. E., \& Shephard, R. J. (2007). Evidence-based guidelines for physical activity of adult Canadians. Applied Physiology, Nutrition, and Metabolism, 32(2), S17-S74.

Wlid, S., Roglic, G., Gree, A., Sicree, R., \& King, H. (2004). Global prevalence of diabetes: Estimates for the year 2000 and projections for 2030. Diabetes Care, 27(5), 1047-1053. 